\title{
Baseline echocardiographic assessment of left ventricle kinetics alteration and mortality risk in a cohort of critically ill COVID-19 patients
}

Davide Ceccato ( $\square$ ceccatodavide@gmail.com )

Università degli studi di Padova

\section{Beatrice Gusella}

Università degli Studi di Padova

Mattia Grassi

University of Padua Medical School

Alessandro Toffolon

University of Padua Medical School

Anna Postal

University of Padua Medical School

Davide Gorgi

University of Padua Medical School

\section{Federico Capone}

Università degli Studi di Padova

\section{Alois Saller}

University of Padua Medical School

\section{Alberto Cipriani}

Università degli Studi di Padova

\section{Cristiano Sarais}

Department of Cardio-Thoraco-Vascular Sciences and Public Health

\section{Roberto Vettor}

Università degli Studi di Padova

\section{Raffaele Pesavento}

Università degli Studi di Padova

Keywords: COVID-19, echocardiography, cardiovascular disease, mortality

Posted Date: June 19th, 2020

DOl: https://doi.org/10.21203/rs.3.rs-35798/v1 
License: (c) (i) This work is licensed under a Creative Commons Attribution 4.0 International License. Read Full License 


\section{Abstract}

Background SARS-CoV2 infection are frequently associated with cardiovascular manifestations, in particular with symptomatic acute coronary syndromes, cardiac arrhythmias and acute heart failure. However, the elevation of serum troponin seems to be non specific, and a cardiologic diagnostic workup should be performed. We aimed to assess the clinical characteristic and the prevalence of left ventricular (LV) dyssynergy patterns in a cohort of hospitalized non-critically ill COVID-19 patients

Methods Consecutive patients with an objective diagnosis of COVID-19, from February to April 2020. Baseline characteristics and comorbidities was collected. In case of increased troponin levels or symptoms suggestive for a concomitant cardiac syndrome, patients undergo to serial electrocardiograms, serial Troponin tests and bedside transthoracic echocardiogram.

Results 402 consecutive patients were enrolled: 55 patients underwent an echocardiographic exam because of an increase in troponin levels or a suspected myocardial injury. Segmental left ventricular abnormalities were found in 10 (median WMSI 2.03 IQR 1.38-2.75) with a median LV ejection fraction was $30.1 \%$ IQR, median troponin level was $3083 \mathrm{ng} / \mathrm{L}$, median BNP was $761 \mathrm{ng} / \mathrm{L}$. Death for any cause occurred in 4 patients among patients with regional LV abnormalities and in 3 with normal regional function $(p=0,02)$.

Discussion A single bedside transthoracic echocardiogram performed in non critically ill COVID-19 patients with suspected cardiac injury has the potential to better assist clinicians in their challenging decision process. As an isolated increase of troponin levels is common in COVID patients, a bed-side echocardiographic evaluation of cardiac function should be routinely implemented during their early evaluation.

\section{Background}

Coronavirus disease 2019 (COVID-19) is a recognized infectious disease that has spread since December 2019, rapidly through-out Wuhan, China, to several countries around the world [1]. Cardiovascular manifestations induced by viral infection has generated considerable concern. Several case series studies have shown that a variable increase of serum troponins levels is common in COVID-19 patients [2-4]. In a not negligible proportion of them, these is associated with symptomatic acute coronary syndromes, cardiac arrhythmias and acute heart failure and lead to worse and more often fatal course of the viral disease, unlike what has been observed in COVID-19 patients with known cardiovascular disease but without troponin elevation [3;5]. In particular, the association between isolated elevated levels of troponin and a viral myocarditis or ischemic cardiac injury remains controversial as many COVID-19 patients cannot receive further cardiologic diagnostic workup (e.g. echocardiography, coronary angiography, cardiac RM). Therefore, we aimed at assessing the clinical characteristic and the prevalence of left ventricular (LV) dyssynergy patterns in a cohort of hospitalized non-critically ill COVID-19 patients with and without increase of troponin levels. 


\section{Methods}

For these purpose we included consecutive patients older than 18 years, with an objective diagnosis of COVID-19-pneumonia admitted to our medical COVID Unit at the University Hospital of Padua, from February to April 2020. Baseline characteristics and comorbidities was collected. At time of the admission, in all patients an electrocardiogram and a set of lab tests, including serum troponin I and Ddimer levels were performed. In case of increased troponin levels or symptoms suggestive for a concomitant cardiac syndrome, patients undergo to serial electrocardiograms, serial Troponin tests, and bedside transthoracic echocardiogram. The left ventricular regional segmental thickening were estimated according to the ASE recommendations for use in clinical trials. Baseline characteristics, echocardiographic and biohumoral parameters were presented using descriptive statistics. Continuous variables were shown as median (IQR) and categorical variables as frequencies and percentages. Comparative analyses were performed using Fisher's exact test.

\section{Results}

Overall, we retrieved data from 402 consecutive patients. Of them, 23 were excluded because of the need for intensive care or ventilatory support at time of admission. Of the remaining 379,55 patients (14.5\%) underwent an echocardiographic exam because of an increase in troponin levels or a suspected myocardial injury. The median age was 71 years and 35 were male (63.6\%). Table 1 summarized the baseline and clinical characteristic of patients. At least one cardiovascular risk factor was present in 47 patients (85.5\%), in particular hypertension (67.3\%), atrial fibrillation (37\%) and history of coronary artery disease (25.5\%). Of the 55 patients (Table 2), segmental left ventricular abnormalities were found in 10 (median WMSI 2.03 IQR 1.38-2.75) and normal regional function in the remaining 45. In patients with regional LV abnormalities, the median LV ejection fraction was $30.1 \%$ IQR, median troponin level was $3083 \mathrm{ng} / \mathrm{L}$, median BNP was $761 \mathrm{ng} / \mathrm{L}$. The corresponding values for patients with normal LV regional function were $57.8 \%, 620 \mathrm{ng} / \mathrm{L}$ and $376 \mathrm{ng} / \mathrm{L}$, respectively. Electrocardiographic finding suggestive of ischemia were found in 21 subjects. 7 patients (70\%) with segmental LV abnormalities and 14 with normal regional function (31\%). Death for any cause occurred in 4 patients among patients with regional LV abnormalities and in 3 with normal regional function $(p=0,02)$. There were no statistically significant differences between the two groups in all other variables analysed, except for history of coronary artery disease $(p=0,01)$. None patients with LV kinetic alteration underwent coronary angiography or percutaneous coronary intervention.

\section{Discussion}

In patients with COVID-19 pneumonia, several symptoms and signs and concomitant laboratory and electrocardiographic abnormalities suggesting or mimicking an acute coronary syndrome are common. However, often suspected cases do not undergo further cardiological workup, because of the concomitant clinical severity of the COVID pneumonia and logistic difficulties in transferring to and undergo coronary angiography or CT scanning. In our study the COVID patients with suspected myocardial injury and an 
abnormal regional LV function had a higher prevalence of severe LV systolic dysfunction, higher levels of troponin and other markers of heart failure and prothrombotic and inflammatory state and higher mortality risk. Furthermore, they carried out more often several cardiovascular risk factors, especially history of coronary artery disease and peripheral artery disease. These findings were in keeping with Shi et al [7] and Guo et al [5] that report similar characteristics of patients who develop myocardial injury and an increase risk of complication. Interestingly enough, in a not negligible proportion of COVID patients with suspected cardiac injury a normal LV regional function, a less severe LV pump function and a trend toward a reduced mortality were found. In addiction, recently Li et al [8] reported a prognostic value of right ventricle dysfunction, providing additive predictive value of echocardiographic examination in COVID-cohort. Our results are of potential relevance as they could help identifying both patients who deserve an non deferrable cardiologic diagnostic workup and those in whom a postponed workup can be at least considered. From these brief observation, we suggest a single bedside transthoracic echocardiogram performed in non critically ill COVID-19 patients with suspected cardiac injury has the potential to better assist clinicians in their challenging decision process. As an isolated increase of troponin levels is common in COVID patients, a bed-side echocardiographic evaluation of cardiac function should be routinely implemented during their early evaluation.

\section{Declarations}

Study data and clinical information were collected and managed by medical staff using REDCap electronic data capture tools hosted at University of Padova. The study protocol was approved by the cardiovascular section of Ethics Committee on Human Research of the Padua. Verbal or written informed consent was obtained from patients surviving hospitalization. The study was performed according to the ethical guidelines of the Declaration of Helsinki (seventh revision).

\section{References}

1. Zhu N, Zhang D, Wang W. et Al. A Novel Coronavirus From Patients With Pneumonia in China, 2019. N Engl J Med. 2020 Feb 20;382(8):727-733. doi: 10.1056/NEJMoa2001017. Epub 2020 Jan 24.

2. Yang $X, Y u Y, X u J$ et Al. Clinical course and outcomes of critically ill patients with SARS-CoV-2 pneumonia in Wuhan, China: a single-centered, retrospective, observational study. Lancet Respir Med 2020 May;8(5):475-481. doi: 10.1016/S2213-2600(20)30079-5. Epub 2020 Feb 24

3. Bonow RO, Fonarow GC, O'Gara PT, Yancy CW. Association of Coronavirus Disease 2019 (COVID-19) With Myocardial Injury and Mortality. JAMA Cardiol. 2020 Mar 27. doi: 10.1001/jamacardio.2020.1105. Online ahead of print.

4. Guo T, Fan Y, Chen M, Wu X, Zhang L, He T, Wang H, Wan J, Wang X, Lu Z. Cardiovascular Implications of Fatal Outcomes of Patients With Coronavirus Disease 2019 (COVID-19). JAMA Cardiol. 2020 Mar 27:e201017. doi: 10.1001/jamacardio.2020.1017. Online ahead of print

5. Peng QY, Wang XT, Zhang LN.Using echocardiography to guide the treatment of novel coronavirus pneumonia. Chinese Critical Care Ultrasound Study Group (CCUSG). Version 2. Crit Care. 2020 Apr 
10;24(1):143. doi: 10.1186/s13054-020-02856-z.

6. Shi S, Qin M, Shen B, Cai Y, Liu T, Yang F, Gong W, Liu X, Liang J, Zhao Q, Huang H, Yang B, Huang C. Association of Cardiac Injury With Mortality in Hospitalized Patients With COVID-19 in Wuhan, China. JAMA Cardiol. 2020 Mar 25:e200950. doi: 10.1001/jamacardio.2020.0950. Online ahead of print.

7. Violi F, Cangemi R, Falcone M, Taliani G, Pieralli F, Vannucchi V, Nozzoli C, Venditti M, Chirinos JA, Corrales-Medina VF. Cardiovascular Complications and Short-term Mortality Risk in CommunityAcquired Pneumonia. For SIXTUS (Thrombosis-Related Extrapulmonary Outcomes in Pneumonia) Study Group. Clin Infect Dis. 2017 Jun 1;64(11):1486-1493. doi: 10.1093/cid/cix164.

8. Li Y, Li H, Zhu S, Xie Y, Wang B et Al.. Prognostic Value of Right Ventricular Longitudinal Strain in Patients with COVID-19. JACC Cardiovasc Imaging. 2020 Apr 28 doi: 10.1016/j.jcmg.2020.04.014

\section{Tables}

Table 1

\begin{tabular}{lc}
\hline Baseline characteristics (N=55) \\
\hline Age (years, av) & $71, \mathrm{SD} 12$ \\
\hline Male (\%) & $35(63,6 \%)$ \\
Caucasian & $52(96.7 \%)$ \\
\hline BMI (kg/m $\left.{ }^{2}\right)$ & $27,4 \mathrm{~kg} / \mathrm{m}^{2}(\mathrm{av}), \mathrm{SD} 4,5$ \\
Hospitalization median (days) & 16,9 days, SD 12,8 days \\
Symphtoms at hospital admission & \\
Fever & $47(85.7 \%)$ \\
Cough & $29(51.8 \%)$ \\
Chest pain & $17(31.6 \%)$ \\
Dyspnoea & $31(57.1 \%)$ \\
\hline
\end{tabular}


Table 2

\begin{tabular}{|c|c|c|c|}
\hline & Alterations $(n=10)$ & No alterations $(n=45)$ & p-value \\
\hline \multicolumn{4}{|l|}{ Risk factors } \\
\hline Atrial fibrillation $n(\%)$ & $3(30)$ & $16(38)$ & 0,728 \\
\hline Coronary artery disease $\mathrm{n}(\%)$ & $6(60)$ & $7(17)$ & 0,01 \\
\hline Diabetes mellitus n (\%) & $4(40)$ & $10(23)$ & 0,428 \\
\hline Dyslipidaemia $n(\%)$ & $4(40)$ & $13(31)$ & 0,711 \\
\hline Hypertension n (\%) & $8(80)$ & $26(86)$ & 0,462 \\
\hline Active smoke $\mathrm{n}(\%)$ & $2(20)$ & $10(24)$ & 1 \\
\hline Chronic kidney injury $\mathrm{n}(\%)$ & $2(20)$ & $1(2)$ & 0,09 \\
\hline Active cancer $\mathrm{n}(\%)$ & $3(30)$ & $7(17)$ & 0,382 \\
\hline Stroke history $n(\%)$ & $2(20)$ & $3(7)$ & 0,242 \\
\hline Peripheral artery disease n (\%) & $4(40)$ & $6(14)$ & 0,084 \\
\hline \multicolumn{4}{|l|}{ Echocardiographic 2D findings } \\
\hline Ejection fraction (\%) & 30.1 & 57.8 & - \\
\hline Wall motion score index & 2.03 & 1.04 & - \\
\hline Regional dissinergic segments & 63.75 & 0 & - \\
\hline ECG alterations $\mathbf{n}(\%)(n=21)$ & $7(33)$ & $14(67)$ & - \\
\hline \multicolumn{4}{|l|}{ Laboratory } \\
\hline Troponin I (ng) & 3083 av (16-17162), & 620 av (26-715) & - \\
\hline Brain Natriuretic Peptide (ng) & 761 av (38-1474), & 376 av (10-1911) & - \\
\hline D-dimer (ng) & 8620 av $(150-65674)$ & 2932 av $(150-36197)$ & - \\
\hline Death n (\%) (n=7) & $4(57)$ & $3(42)$ & 0,019 \\
\hline
\end{tabular}

